\title{
5D Attractors with Higher Derivatives
}

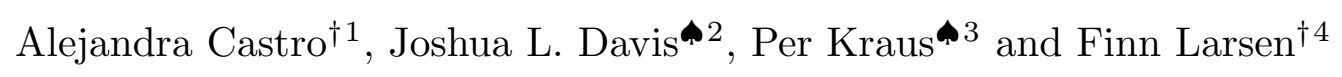 \\ $\dagger$ 'Michigan Center for Theoretical Physics, Department of Physics \\ University of Michigan, Ann Arbor, MI 48109-1120, USA. \\ - Department of Physics and Astronomy, UCLA, \\ Los Angeles, CA 90095-1547, USA.
}

\begin{abstract}
We analyze higher derivative corrections to attractor geometries in five dimensions. We find corrected $\mathrm{AdS}_{3} \times S^{2}$ geometries by solving the equations of motion coming from a recently constructed four-derivative supergravity action in five dimensions. The result allows us to explicitly verify a previous anomaly based derivation of the $\mathrm{AdS}_{3}$ central charges of this theory. Also, by dimensional reduction we compare our results with those of the $4 \mathrm{D}$ higher derivative attractor, and find complete agreement.
\end{abstract}

February, 2007

\footnotetext{
1 aycastro@umich.edu

2 davis@physics.ucla.edu

3 pkraus@ucla.edu

4 larsenf@umich.edu
} 


\section{Introduction}

The last few years have seen progress in our understanding of corrections to the entropy of black holes in string theory, both at the microscopic and macroscopic levels. On the supergravity side, this has meant studying the effect of higher derivative terms in the action [1,2, 3, 1 . 4D extremal black holes have a near horizon $\mathrm{AdS}_{2} \times S^{2}$ geometry, with moduli fixed by the attractor mechanism [5]. By using the corrected attractor solution and the general Wald entropy formula [6], it is possible to successfully match an infinite series of corrections to the Bekenstein-Hawking area law with the corresponding microscopic degeneracy of states [1, 2, 3, 3, 7,8,9,10, 11, 12]. For reviews see [13, 14, 15].

However, on closer inspection this success actually seems quite mysterious, since only a selected subset of terms in the supergravity action are being used. Namely, one incorporates the supersymmetric completion of certain $R^{2}$ terms (as can be captured by corrections to the generalized prepotential), but neglects various $R^{4}$ and higher order terms, even though these a priori contribute at the order one is working. There is at present no $4 \mathrm{D}$ understanding of why these terms can be neglected.

Greater control is achieved by realizing that these black holes admit near horizon $\mathrm{AdS}_{3} \times S^{2}$ geometries [3]. To relate an $\mathrm{AdS}_{2} \times S^{2}$ geometry to $\mathrm{AdS}_{3} \times S^{2}$, one interprets one of the $4 \mathrm{D}$ gauge fields as coming from a Kaluza-Klein circle [16]. An $\mathrm{AdS}_{3} \times S^{2}$ region then appears provided that there is vanishing Kaluza-Klein monopole charge $\left(p^{0}=0\right)$. Alternatively, one can study these black holes in the context of 5D supergravity.

By using the extra symmetries inherent in the 5D near horizon description, one finds that the corrected entropy formula is governed by the coefficients of the Chern-Simons terms in the supergravity action. In this way it is possible to bypass the need to find the full set of higher derivative terms, or to find the explicit values of the near horizon

moduli. The key observation is that the entropy formula is controlled by the values of the left and right moving central charges of the associated $1+1$ dimensional CFT, and due to supersymmetry these are completely determined by gauge and gravitational anomalies.

To verify this picture explicitly, and to find the corrected black hole geometry, one needs to work with the full 5D susy invariant four derivative action, corresponding to the supersymmetric completion of the four derivative Chern-Simons terms. This action appeared recently in [17]. In this paper we find the near horizon $\mathrm{AdS}_{3} \times S^{2}$ geometry by analyzing the BPS conditions and the equations of motion coming from this action. To do this, it is most efficient to employ the " $c$-extremization" procedure developed in [3] (or the closely related "entropy-function" developed in [4]). The strategy is to write down a c-function whose critical points correspond to the solutions of the equations of motion. Furthermore, the value of the c-function at a critical point is equal to the average of the left and right moving central charges of the associated CFT. We will show that the result is in precise agreement with the values inferred from the supersymmetry/anomaly based 
argument, and thereby verify that the entropy is indeed controlled by the Chern-Simons terms. More generally, this same logic leads to the conclusion that there are no further corrections to the central charges from additional higher derivative terms (i.e. more than four derivatives), since we have already taken into account the full set of terms related by supersymmetry to the Chern-Simons terms.

Since this procedure also yields the values of the fixed 5D moduli, we can compare with the known results for the 4D moduli. Writing out the details of the reduction from $5 \mathrm{D}$ to $4 \mathrm{D}$ we find full agreement.

This article is organized as follows. In section 2 we review c-extremization. In section 3 we illustrate the procedure for the leading order action. In section 4 we analyze the supersymmetry conditions in an off-shell form that applies also when higher derivatives are taken into account. In section 5 we carry out the c-extremization procedure on the full action including all terms that are related to the Chern-Simons term by supersymmetry. We find results that are consistent with the supersymmetry conditions from section 4 and moreover find a central charge that agrees with the one previously found using supersymmetry and anomalies. In section 6 we compare our results with those found for black holes in four dimensions and find complete agreement.

\section{Review of c-extremization}

The problem of finding an $\mathrm{AdS}_{3} \times S^{2}$ solution 5 to a general higher derivative action can be reduced to the problem of extremizing a single function of the scale sizes and moduli [3]. Furthermore, the value of this function at its critical point is (after suitable normalization) equal to the average of the left and right moving central charges of the asymptotic conformal symmetry group of the theory. In this section we review how this works.

We look for a solution respecting all $\mathrm{AdS}_{3} \times S^{2}$ isometries. Besides constant scalar fields, we can also have two-form fields proportional to the volume form on $S^{2}$. More generally, we could also have three-form fields proportional to the $\mathrm{AdS}_{3}$ volume form; these will not make any appearance in this paper, but we note that they would necessitate a modification of some of the following formulas.

Let the action for the theory be of the form

$$
S=\frac{1}{4 \pi^{2}} \int d^{5} x \sqrt{g} \mathcal{L}+S_{C S}+S_{\mathrm{bndy}}
$$

\footnotetext{
${ }^{5}$ Or more generally an $\mathrm{AdS}_{p} \times S^{q}$ solution
} 
with $G_{5}=\frac{\pi}{4}$. Our trial solution takes the form

$$
\begin{aligned}
d s^{2} & =\ell_{A}^{2} d s_{A d S}^{2}+\ell_{S}^{2} d \Omega_{2}^{2} \\
F^{I} & =\frac{p^{I}}{2} \epsilon_{2} \\
v & =V \epsilon_{2} \\
\phi^{a} & =\text { constant } .
\end{aligned}
$$

Here $F^{I}$ denote two-form fields strengths with magnetic charges $p^{I} ; v$ denotes additional two-form field(s); and $\phi^{a}$ denote physical and auxiliary scalar fields. $\epsilon_{2}$ is the volume form on the unit $S^{2}$. We normalize the $F^{I}$ such that the charges $p^{I}$ are integer quantized.

Since all covariant derivatives are assumed to vanish, the equations of motion following from (2.1), evaluated on the trial solution (2.2), reduce to extremizing the function $\sqrt{g} \mathcal{L}$ as a function of $\ell_{A}, \ell_{S}, V$ and $\phi^{a}$. Equivalently, we can extremise the c-function, defined as

$$
c=-6 \ell_{A}^{3} \ell_{S}^{2} \mathcal{L}
$$

When extremizing, we hold fixed the quantized charges $p^{I}$, so that all free parameters are determined in terms of the $p^{I}$ (or else are undetermined). This is the attractor mechanism, fixing the geometry and moduli in terms of the charges.

The choice of normalization in (2.3) is motivated as follows. The theory on $\mathrm{AdS}_{3}$ has a boundary stress tensor [18] whose trace anomaly is [19]

$$
T_{i}^{i}=-\frac{c}{12} R^{(2)}
$$

where $R^{(2)}$ is the scalar curvature of the conformal boundary metric. The prefactor in (2.3) was chosen such that the c-function evaluated at its critical point is equal to the $c$ appearing in (2.4). In a theory with equal left and right moving central charges, $c$ is the central charge. More generally, the trace anomaly is related to the average:

$$
c=\frac{1}{2}\left(c_{L}+c_{R}\right)
$$

Given the central charges, evaluation of the Euclidean black hole action leads to the general formula for the black hole entropy $s$ :

$$
s=2 \pi \sqrt{\frac{c_{L}}{6}\left(L_{0}-\frac{c_{L}}{24}\right)}+2 \pi \sqrt{\frac{c_{R}}{6}\left(\tilde{L}_{0}-\frac{c_{R}}{24}\right)} .
$$

Since this expression takes the same form as Cardy's formula it is convenient for comparison with microscopic results. Here, however, it is just a statement about the on-shell 
supergravity action. In fact, for $c_{L}=c_{R}(2.6)$ agrees with Wald's entropy formula independently of the microscopic theory [20, 3] 6 .

We will be working with five dimensional supergravity coupled to vector multiplets, which can be thought of as arising from M-theory compactified on $C Y_{3}$. The charges $p^{I}$ then correspond to M5-branes wrapping 4-cycles in $C Y_{3}$. The central charges are known to be [22,23]

$$
c_{L}=6 p^{3}+\frac{1}{2} c_{2} \cdot p, \quad c_{R}=6 p^{3}+c_{2} \cdot p
$$

with

$$
p^{3}=\frac{1}{6} c_{I J K} p^{I} p^{J} p^{K}
$$

where $c_{I J K}$ are the triple intersection numbers of the $C Y_{3}$, and $c_{2 I}$ are the expansion coefficients of the second Chern class. This then gives the following prediction for the extremal value of the c-function

$$
c=6 p^{3}+\frac{3}{4} c_{2} \cdot p
$$

This is the result we wish to verify from the explicit higher derivative action.

\section{Two derivative analysis}

\subsection{Five dimensional off-shell supergravity.}

Following [17] (see [24,25] for earlier work) we consider superconformal gravity in five dimensions. The local form of the theory is off-shell, meaning that the auxiliary fields in the multiplets are not integrated out. At two-derivative order the bosonic terms in the Lagrangian are

$$
\begin{aligned}
\frac{1}{2} \mathcal{L}_{0}= & \partial^{a} \mathcal{A}_{i}^{\alpha} \partial_{a} \mathcal{A}_{\alpha}^{i}+\mathcal{A}^{2}\left(\frac{1}{8} D-\frac{3}{16} R-\frac{1}{4} v^{2}\right) \\
& +\mathcal{N}\left(\frac{1}{4} D+\frac{1}{8} R+\frac{3}{2} v^{2}\right)+\mathcal{N}_{I} v^{a b} F_{a b}^{I} \\
& +\mathcal{N}_{I J}\left(\frac{1}{8} F_{a b}^{I} F^{J a b}+\frac{1}{4} \partial_{a} M^{I} \partial^{a} M^{J}\right)+\frac{1}{48} e^{-1} c_{I J K} A_{a}^{I} F_{b c}^{J} F_{d e}^{K} \epsilon^{a b c d e}
\end{aligned}
$$

\footnotetext{
$6 \quad$ Theories with $c_{L} \neq c_{R}$ have gravitational Chern-Simons terms that violate diffeomorphism
} invariance so Wald's formula does not apply. [21] generalizes Wald's formula to this case and shows that agreement with (2.6) is maintained.

7 We have omitted fields in the multiplets associated with gauged supergravity or that can be turned off by gauge fixing conformal symmetries. With respect to [17] we have switched the sign in the kinetic term for the scalars $M^{I}$ and the sign of the Ricci scalar $\left(R_{\text {here }}=-R_{\text {there }}\right)$. 
We are taking into account the bosonic fields of two distinct super multiplets: the Weyl multiplet, contains the vielbein $e_{\mu}{ }^{a}$, the two-form auxiliary field $v_{a b}$, and a scalar auxiliary field $D$; the vector multiplets enumerated by index $I=1 \ldots n_{V}$, each containing a one-form gauge field $A^{I}$ and scalar $M^{I}$, with $F^{I}=d A^{I}$. Although we will not discuss gauge fixing in detail, it is useful to include a term for the hyper multiplet which contains the Weyl scalar $\mathcal{A}_{i}^{\alpha}$. The index $i=1,2$ refers to $S U(2)$ doublets and $\alpha=1, \ldots 2 r$ refers to the $U S p(2 r)$. The hyper is used to gauge fix the dilatational symmetry and we choose a gauge that satisfies

$$
\mathcal{A}^{2}=-2, \quad \partial_{a} \mathcal{A}_{\alpha}^{i}=0
$$

The functions on the scalar manifold are defined by

$$
\mathcal{N}=\frac{1}{6} c_{I J K} M^{I} M^{J} M^{K}, \quad \mathcal{N}_{I}=\partial_{I} \mathcal{N}=\frac{1}{2} c_{I J K} M^{J} M^{K}, \quad \mathcal{N}_{I J}=c_{I J K} M^{K}
$$

The auxiliary field $D$ appears linearly in (3.1), which means that it acts as a Lagrange multiplier. The resulting constraint determines $\mathcal{N}$, which can be thought of as the volume of the compactification manifold. Given that we chose $\mathcal{A}^{2}=-2$, solving the equation of motion for $D$ implies $\mathcal{N}=1$. So, at the level of the two derivative action the scalars are described using real special geometry. For a pedagogical introduction see [26].

We can eliminate the auxiliary fields $v_{a b}$ and $D$ by solving their equations of motion. This gives

$$
\mathcal{L}_{0}=-\mathcal{N}\left[-R+G_{I J} \partial_{a} M^{I} \partial^{a} M^{J}+\frac{1}{2} G_{I J} F_{a b}^{I} F^{J a b}-\frac{e^{-1}}{24 \mathcal{N}} c_{I J K} A_{a}^{I} F_{b c}^{J} F_{d e}^{K} \epsilon^{a b c d e}\right]
$$

with

$$
G_{I J}=-\frac{1}{2} \partial_{I} \partial_{J}(\ln \mathcal{N})=\frac{1}{2}\left(\frac{\mathcal{N}_{I} \mathcal{N}_{J}}{\mathcal{N}^{2}}-\frac{\mathcal{N}_{I J}}{\mathcal{N}}\right)
$$

This is the familiar two derivative Lagrangian in five dimensional supergravity. For our purposes, we will not use (3.4) and instead work with (3.1).

\section{2. c-extremization}

We now determine, at the two-derivative order, the near horizon $\operatorname{AdS}_{3} \times S^{2}$ geometry corresponding to a black string in five dimensions, which we will refer to as the "black string attractor". The near horizon configuration is given by (2.2) and the central charge as defined in $(2.3)$ is

$$
c=-6 \ell_{A}^{3} \ell_{S}^{2} \mathcal{L}_{0}
$$


with $\mathcal{L}_{0}$ given by (3.1) evaluated on the trial solution (2.2). For this configuration, we will have some simplifications. The Chern-Simons term in (3.1) vanishes and derivatives of the scalars $M^{I}$ are zero. The Ricci scalar is

$$
R=-\frac{6}{\ell_{A}^{2}}+\frac{2}{\ell_{S}^{2}} .
$$

By symmetry, we know that the scalars $M^{I}$ are proportional to the charges $p^{I}$, so we write $M^{I}=m p^{I}$, with $m$ a constant to be determined. The $c$-function then becomes,

$$
\begin{aligned}
c=-12 \ell_{A}^{3} \ell_{S}^{2}( & \frac{1}{4}\left(p^{3} m^{3}-1\right) D-\frac{1}{4}\left(p^{3} m^{3}+3\right)\left(\frac{3}{\ell_{A}^{2}}-\frac{1}{\ell_{S}^{2}}\right) \\
+ & \left.\frac{1}{\ell_{S}^{4}}\left(\left(3 p^{3} m^{3}+1\right) V^{2}+3 p^{3} m^{2} V\right)+\frac{3 p^{3}}{\ell_{S}^{4}} \frac{m}{8}\right),
\end{aligned}
$$

with $p^{3}$ given by equation (2.8). Extremizing (3.8) with respect to $D$ imposes $m^{3}=p^{-3}$ and the equation for $V$ gives

$$
V=-\frac{3}{8} p
$$

The extremization of 3.8 with respect to the radii $\ell_{A}$ and $\ell_{S}$ results in

$$
\ell_{A}=2 \ell_{S}, \quad \ell_{A}=p
$$

Finally, by extremizing the $c$-function with respect to $m$ we find $D=12 p^{-2}$. Summarizing, our result for the parameters of the solution is

$$
M^{I}=\frac{p^{I}}{p}, \quad \ell_{A}=2 \ell_{S}=p, \quad D=\frac{12}{p^{2}}, \quad V=-\frac{3 p}{8} .
$$

Inserting (3.11) in (3.8), the central charge for the black string in the two derivative theory is

$$
c=6 p^{3}=c_{I J K} p^{I} p^{J} p^{K} .
$$

The value of $c$ agrees with the expectation (2.9) to the leading order in charges. The new feature is verifying that $c$-extremization off-shell (i.e. keeping auxiliary fields) is consistent.

\section{Susy variations}

We would like to determine corrections to the attractor solution from the higher derivative terms in the action. A strong constraint comes from the fact that the attractor 
solution exhibits maximal supersymmetry. Furthermore, in the off-shell formulation the supersymmetry transformations are independent of the detailed form of the action (i.e. they are the same for the two and four derivative actions). With this in mind, we now analyze the constraints from supersymmetry.

The supersymmetry variations are

$$
\begin{aligned}
\delta \psi_{\mu}^{i} & =\mathcal{D}_{\mu} \varepsilon^{i}+\frac{1}{2} v^{a b} \gamma_{\mu a b} \varepsilon^{i}-\gamma_{\mu} \eta^{i} \\
\delta \chi^{i} & =D \varepsilon^{i}-2 \gamma^{c} \gamma^{a b} \varepsilon^{i} \mathcal{D}_{a} v_{b c}-2 \gamma^{a} \varepsilon^{i} \epsilon_{a b c d e} v^{b c} v^{d e}+4 \gamma \cdot v \eta^{i}, \\
\delta \Omega^{I i} & =-\frac{1}{4} \gamma \cdot F^{I} \varepsilon^{i}-\frac{1}{2} \gamma^{a} \partial_{a} M^{I} \varepsilon^{i}-M^{I} \eta^{i} \\
\delta \zeta^{\alpha} & =\gamma^{a} \partial_{a} \mathcal{A}_{j}^{\alpha} \varepsilon^{j}-\gamma \cdot v \varepsilon^{j} \mathcal{A}_{j}^{\alpha}+3 \mathcal{A}_{j}^{\alpha} \eta^{j} .
\end{aligned}
$$

The first two transformations come from the fermions in the Weyl multiplet, the gravitino $\psi_{\mu}^{i}$ and an auxiliary Majorana spinor $\chi^{i}$. From the vector multiplets we have the gaugino $\Omega_{i}^{I}$ and the hyper multiplet contributes with $\zeta^{\alpha}$. We are using the notation $\gamma \cdot v=\gamma_{a b} v^{a b}$.

\subsection{Supersymmetry constraints for the black string attractor.}

The supersymmetry transformations (4.1) simplify dramatically when evaluated on our trial background (2.2). The attractor has maximal supersymmetry, meaning all variations must vanish. In our background this reduces to solving

$$
\begin{aligned}
\delta \psi_{\mu}^{i} & =\mathcal{D}_{\mu} \varepsilon^{i}+\frac{1}{2} v^{a b} \gamma_{\mu a b} \varepsilon^{i}-\gamma_{\mu} \eta^{i}=0, \\
\delta \chi & =D \varepsilon^{i}+4 \gamma \cdot v \eta^{i}=0, \\
\delta \Omega^{I i} & =-\frac{1}{4} \gamma \cdot F^{I} \varepsilon^{i}-M^{I} \eta^{i}=0, \\
\delta \zeta^{\alpha} & =\left(-\gamma \cdot v \varepsilon^{j}+3 \eta^{j}\right) \mathcal{A}_{j}^{\alpha}=0 .
\end{aligned}
$$

From the gaugino variation it is clear that the scalars $M^{I}$ are proportional to the charges $p^{I}$, so we can write $M^{I}=m p^{I}$, where the constant of proportionality $m$ will be determined by the remaining equations. The last equation in (4.2) gives

$$
\eta^{i}=\frac{1}{3} \gamma \cdot v \varepsilon^{i}
$$

Inserting (4.3) in (4.2) we get for the gravitino variation

$$
\left(\mathcal{D}_{\mu}+\frac{1}{2} v^{a b} \gamma_{\mu a b}-\frac{1}{3} v^{a b} \gamma_{\mu} \gamma_{a b}\right) \varepsilon^{i}=0
$$


and for the auxiliary field and gaugino

$$
\begin{aligned}
\left(D+\frac{4}{3}(\gamma \cdot v)^{2}\right) \varepsilon^{i} & =0 \\
\left(-\frac{1}{4} \gamma \cdot F^{I}-\frac{m}{3} p^{I} \gamma \cdot v\right) \varepsilon^{i} & =0 .
\end{aligned}
$$

Solving (4.5) on the ansatz (2.2), we find

$$
m V=-\frac{3}{8}, \quad D=\frac{16}{3} \frac{V^{2}}{\ell_{S}^{4}} .
$$

Finally, from the gravitino variation 4.4 ) 8 we get a relation between the radii, $\ell_{A}$ and $\ell_{S}$, and the auxiliary field $V$

$$
\ell_{A}=2 \ell_{S}, \quad V=-\frac{3}{8} \ell_{A}
$$

The relations between the moduli shown in equations (4.6) and (4.7) hold independently of the action. Since the supersymmetry variations are exact off-shell, these results will not change for higher derivatives theories.

It is important to note that supersymmetry does not fully determine the values of the moduli and as presented here, one of the fields is unconstrained. This should be expected, since there are gauge symmetries unrelated to supersymmetry transformations that we have not imposed. For example, in the leading order theory described by (3.1), the scalars are described using real special geometry, where the volume $\mathcal{N}$ is fixed. This comes about from fixing the superconformal theory to Poincare supergravity using the equation of motion for $D$, and is not related to the fact that the theory is supersymmetric. When higher derivatives terms are included in the theory, one should similarly expect to use at least one equation of motion from the off-shell theory to specify the solution completely.

Without loss of generality, we will take the $A d S_{3}$ radius as the undetermined modulus, and so we can summarize our results as

$$
\ell_{S}=\frac{1}{2} \ell_{A}, \quad m=\frac{1}{\ell_{A}}, \quad V=-\frac{3}{8} \ell_{A}, \quad D=\frac{12}{\ell_{A}^{2}} .
$$

Comparing with the two derivative $c$-extremization, we can see that (4.8) agrees with (3.11). The piece of information that is missing from the supersymmetry constraints is the relation between $\ell_{A}$ and the charges $p^{I}$. At the level of the two derivative theory, this is simply $\ell_{A}=p$. As we will show in the next section, when higher derivatives are taken into account, the $A d S_{3}$ radius will be modified and the value of the corrected moduli will be determined by our procedure.

8 See [27], [28] and references therein for details on manipulation of the gravitino variation. 


\section{5. c-extremization including higher derivatives}

We are now ready to discuss higher derivative corrections to the central charge. As mentioned in the introduction, we want to verify that the Chern-Simons term controls the corrections to the central charge. From anomaly arguments, this term is given by

$$
\sqrt{g} \mathcal{L}_{C S}=-\frac{c_{2 I}}{48 \cdot 2} A^{I} \wedge \operatorname{Tr}(R \wedge R)=\frac{c_{2 I}}{24 \cdot 16} \epsilon_{a b c d e} A^{I a} R^{b c f g} R_{f g}^{d e} .
$$

The Chern-Simons term by itself is not supersymmetric and therefore extra terms should be included. The four derivative supersymmetric completion of (5.1) was computed in [17], and the relevant terms for our discussion are

$$
\begin{aligned}
\mathcal{L}_{1}=\frac{c_{2 I}}{24}( & \frac{1}{16} e^{-1} \epsilon_{a b c d e} A^{I a} C^{b c f g} C^{d e}{ }_{f g}+\frac{1}{8} M^{I} C^{a b c d} C_{a b c d}+\frac{1}{12} M^{I} D^{2}+\frac{1}{6} F^{I a b} v_{a b} D \\
& -\frac{1}{3} M^{I} C_{a b c d} v^{a b} v^{c d}-\frac{1}{2} F^{I a b} C_{a b c d} v^{c d}+\frac{8}{3} M^{I} v_{a b} \hat{\mathcal{D}}^{b} \hat{\mathcal{D}}_{c} v^{a c} \\
& +\frac{4}{3} M^{I} \hat{\mathcal{D}}^{a} v^{b c} \hat{\mathcal{D}}_{a} v_{b c}+\frac{4}{3} M^{I} \hat{\mathcal{D}}^{a} v^{b c} \hat{\mathcal{D}}_{b} v_{c a}-\frac{2}{3} e^{-1} M^{I} \epsilon_{a b c d e} v^{a b} v^{c d} \hat{\mathcal{D}}_{f} v^{e f} \\
& +\frac{2}{3} e^{-1} F^{I a b} \epsilon_{a b c d e} v^{c d} \hat{\mathcal{D}}_{f} v^{e f}+e^{-1} F^{I a b} \epsilon_{a b c d e} v^{c} \hat{\mathcal{D}}^{d} v^{e f} \\
& \left.-\frac{4}{3} F^{I a b} v_{a c} v^{c d} v_{d b}-\frac{1}{3} F^{I a b} v_{a b} v^{2}+4 M^{I} v_{a b} v^{b c} v_{c d} v^{d a}-M^{I}\left(v_{a b} v^{a b}\right)^{2}\right)
\end{aligned}
$$

with $C_{a b c d}$ the Weyl tensor defined as

$$
C_{c d}^{a b}=R^{a b}{ }_{c d}+\frac{1}{6} R \delta_{[c}^{[a} \delta_{d]}^{b]}-\frac{4}{3} \delta_{[c}^{[a} R_{d]}^{b]} .
$$

The double covariant derivative of $v_{a b}$ has curvature contribution $\$ 9$ given by

$$
v_{a b} \hat{\mathcal{D}}^{b} \hat{\mathcal{D}}_{c} v^{a c}=v_{a b} \mathcal{D}^{b} \mathcal{D}_{c} v^{a c}+\frac{2}{3} v^{a c} v_{c b} R_{a}^{b}+\frac{1}{12} v_{a b} v^{a b} R
$$

\subsection{Central Charge and Moduli Corrections}

Using the $c$-extremization procedure explained in section 2, we will find the corrected central charge and moduli in the higher derivative theory for the $5 D$ black string. Including the four derivative Lagrangian, the central charge becomes

$$
c=-6 \ell_{A}^{3} \ell_{S}^{2}\left(\mathcal{L}_{0}+\mathcal{L}_{1}\right)
$$

9 The sign difference with respect to [17] is coming from the difference in curvature convention. 
where $\mathcal{L}_{0}$ and $\mathcal{L}_{1}$ are given by (3.1) and (5.2) evaluated in the $A d S_{3} \times S^{2}$ background. Using (2.2) and the attractor value for the moduli $M^{I}=m p^{I}$, the supersymmetric four derivative contributions to the central charge are

$$
\begin{aligned}
\mathcal{L}_{1}=\frac{c_{2} \cdot p}{24}[ & \frac{m}{4}\left(\frac{1}{\ell_{A}^{2}}-\frac{1}{\ell_{S}^{2}}\right)^{2}+\frac{2}{3} \frac{V^{3}}{\ell_{S}^{8}}+4 m \frac{V^{4}}{\ell_{S}^{8}}+m \frac{D^{2}}{12}+\frac{D}{6} \frac{V}{\ell_{S}^{4}} \\
& \left.-\frac{2}{3} m \frac{V^{2}}{\ell_{S}^{4}}\left(\frac{3}{\ell_{A}^{2}}+\frac{5}{\ell_{S}^{2}}\right)+\frac{1}{2} \frac{V}{\ell_{S}^{4}}\left(\frac{1}{\ell_{A}^{2}}-\frac{1}{\ell_{S}^{2}}\right)\right] .
\end{aligned}
$$

On our trial background $\hat{\mathcal{D}}_{a} v_{b c}=0$. The two derivative contribution to (5.5) is still given by $(3.8)$.

According to $c$-extremization we should now extremize with respect to all parameters. It would be extremely difficult to do this were it not for the guidance provided by the supersymmetry analysis in the previous section. For example, the variation of (5.5) with respect to $m$ gives

$$
\begin{aligned}
& \frac{3 p^{3} m^{2}}{4}\left(D-\frac{3}{\ell_{A}^{2}}+\frac{1}{\ell_{S}^{2}}\right)+\frac{3 p^{3}}{\ell_{S}^{4}}\left(3 m^{2} V^{2}+2 m V+\frac{1}{8}\right)+ \\
& +\frac{c_{2} \cdot p}{48}\left[\frac{1}{4}\left(\frac{1}{\ell_{A}^{2}}-\frac{1}{\ell_{S}^{2}}\right)^{2}+4 \frac{V^{4}}{\ell_{S}^{8}}+\frac{D^{2}}{12}-\frac{2}{3} \frac{V^{2}}{\ell_{S}^{4}}\left(\frac{3}{\ell_{A}^{2}}+\frac{5}{\ell_{S}^{2}}\right)\right]=0 .
\end{aligned}
$$

It is easy to verify that the moduli given in (4.8) do indeed satisfy this equation.

Before proceeding with the remaining extremization conditions, recall that the BPS conditions (4.2) leave one modulus undetermined. The missing information about the attractor can be simply obtained from the $c$-extremization procedure. The simplest is to consider the equation for the auxiliary field $D$

$$
\frac{\partial c}{\partial D}=0 \quad \Rightarrow \quad p^{3} m^{3}=1-\frac{1}{72} c_{2} \cdot p\left(m D+\frac{V}{\ell_{S}^{4}}\right)
$$

Using (4.8) and solving for the $A d S_{3}$ radius $\left(\ell_{A}\right)$ we find

$$
\ell_{A}^{3}=p^{3}+\frac{1}{12} c_{2} \cdot p
$$

The solution is now fully specified. At this point it is straightforward to vary the c-function (5.5) also with respect to $V, \ell_{A}, \ell_{S}$ and show that the resulting equations are satisfied by (4.8) and (5.9). All in all we have found a solution that extremizes the c-function and shown that this solution is supersymmetric. Since we proceeded somewhat indirectly, we have not excluded the existence of other solutions with the same charge configuration but no supersymmetry. 
Evaluating the c-function for our solution we find the corrected central charge

$$
c=6 p^{3}+\frac{3}{4} c_{2} \cdot p .
$$

This is precisely the result (2.9) that was predicted from supersymmetry and anomalies.

It is worth noting that the simple form of this result comes about in a rather nontrivial way in the present procedure. The radius of curvature $\ell_{A}(5.9)$ is a nontrivial function of the charges and it enters in the denominator of the Lagrangian (5.6). It is only due to intricate cancellations that the final result (5.10) becomes a linear function of the charges $p^{I}$. That this works out correctly provides a rather stringent consistency check on the entire framework.

\subsection{Small black holes}

One of the benefits of considering higher derivative corrections is that we can find smooth solutions in cases where the two-derivative action would yield a naked singularity. These are so-called "small black holes" [8,9]. In particular, if we choose charges $p^{I}$ such that $p^{3}=0$ but $c_{2} \cdot p \neq 0$, then the two-derivatives formulas (3.11) become singular, while the four derivative formulas given in this section are well behaved. We can further check that the full action expanded around the small black hole solution exhibits no obvious pathologies. On the other hand, since some of the moduli $M^{I}$ now vanish, some of the internal compactification cycles are becoming small, and so one should be alert to potentially large corrections from non-perturbative effects not included here. See [9] for more discussion.

\section{Comparison between $5 \mathrm{D}$ and $4 \mathrm{D}$ attractor formulas}

So far we have focussed on the $A d S_{3} \times S^{2}$ attractor geometry near an effective string in five dimensions. Most recent works on higher curvature corrections are in the context of black holes in four dimensions and their $A d S_{2} \times S^{2}$ near horizon attractor geometry. It is instructive to work out the detailed comparison between the four and five dimensional perspective in view of the higher derivative corrections. This is achieved by wrapping the string on a circle and dimensionally reducing. In terms of black hole entropy counting, a recent discussion of the relation between the $\mathrm{AdS}_{3}$ and $\mathrm{AdS}_{2}$ viewpoints can be found in [29].

A good starting point is the $A d S_{3}$ geometry written in Poincare coordinates as

$$
d s_{3}^{2}=\frac{\ell_{A}^{2}}{y^{2}}\left(d w^{+} d w^{-}+d y^{2}\right) .
$$


Introducing the coordinates 30

$$
\begin{aligned}
w^{+} & =\frac{1}{2 \pi T_{L}} e^{2 \pi T_{L}\left(x^{5}+t\right),} \\
w^{-} & =x^{5}-t-\frac{\ell_{A}^{4} \pi T_{L}}{U^{2}}, \\
y & =\frac{\ell_{A}^{2}}{U} e^{\pi T_{L}\left(x^{5}+t\right)},
\end{aligned}
$$

the line element becomes

$$
d s_{3}^{2}=\frac{U^{2}}{\ell_{A}^{2}}\left(d x_{5}^{2}-d t^{2}\right)+\ell_{A}^{2} \frac{d U^{2}}{U^{2}}+\pi^{2} \ell_{A}^{2} T_{L}^{2}\left(d x_{5}+d t\right)^{2} .
$$

Wrapping the string on a circle corresponds to imposing the periodicity condition $x^{5} \equiv$ $x^{5}+2 \pi R$, which amounts to identifications on the $A d S_{3}$ space that change the causal structure to that of a black hole [31]. This is clearest if we introduce the Schwarzschildtype coordinates

$$
\begin{aligned}
\rho^{2} & =\left(\pi^{2} \ell_{A}^{2} T_{L}^{2}+\frac{U^{2}}{\ell_{A}^{2}}\right) R^{2}, \\
x_{5} & =R \phi \\
\tau & =\frac{\ell_{A}}{R} t .
\end{aligned}
$$

Then the line element becomes

$$
d s_{\mathrm{BTZ}}^{2}=-N^{2} d \tau^{2}+N^{-2} d \rho^{2}+\rho^{2}\left(d \phi+N_{\phi} d \tau\right)^{2}
$$

where

$$
\begin{aligned}
N & =\frac{\rho}{\ell_{A}}-\frac{\pi^{2} \ell_{A} T_{L}^{2} R^{2}}{\rho}, \\
N_{\phi} & =\frac{\pi^{2} \ell_{A} T_{L}^{2} R^{2}}{\rho^{2}} .
\end{aligned}
$$

We are interested in the direct product of the three dimensional geometry just introduced and an additional $S^{2}$. Kaluza-Klein reduction on $x^{5}$ takes us from 5D to 4D. The compactification yields a $4 \mathrm{D}$ dilaton with near horizon value

$$
e^{-2 \Phi}=\rho_{\mathrm{hor}}=\pi \ell_{A} T_{L} R
$$

So far we have just reviewed a standard construction. The issue we wish to emphasize is that these considerations are purely geometric and thus hold regardless of the details of the action. The corrections due to higher curvature terms enter only through the relation 
between parameters in the geometry and the underlying microscopic parameters. For example, we found earlier that the $A d S_{3}$ radius is

$$
\ell_{A}=p_{R}
$$

where

$$
p_{R}^{3}=\frac{1}{6}\left(c_{I J K} p^{I} p^{J} p^{K}+\frac{1}{2} c_{2} \cdot p\right) .
$$

We also found the attractor values of the 5D scalars as

$$
X^{I}=\frac{p^{I}}{p_{R}} .
$$

Note that the corrections again appear through $p_{R}$.

We are also interested in the thermodynamics for a black string excited to level $\left|q_{0}\right|$. The corrected formula for the entropy is

$$
S=2 \pi \sqrt{\frac{c_{L}\left|q_{0}\right|}{6}}=2 \pi \sqrt{p_{L}^{3}\left|q_{0}\right|},
$$

where the corrections enter through

$$
p_{L}^{3}=\frac{1}{6}\left(c_{I J K} p^{I} p^{J} p^{K}+c_{2} \cdot p\right)
$$

The energy of the excitations is $E_{L}=\left|q_{0}\right| / R$ and so the first law of thermodynamics gives the temperature

$$
T_{L}=\frac{1}{\pi R} \sqrt{\frac{\left|q_{0}\right|}{p_{L}^{3}}} .
$$

We see again that it is the combination $p_{L}$ that appears in the thermodynamics.

We next consider the corrections of some less obvious quantities. For example, the precise value for the $4 \mathrm{D}$ dilaton (6.7) is

$$
e^{-2 \Phi}=p_{R} \sqrt{\frac{\left|q_{0}\right|}{p_{L}^{3}}} .
$$

The string frame radius of the very near horizon $A d S_{2}$ close to the $4 \mathrm{D}$ black hole is inherited from the $A d S_{3}$ [32] and so its value is simply $\ell_{A}$. The $A d S_{2}$ radius in $4 \mathrm{D}$ Einstein frame is therefore

$$
R_{0}^{2}=e^{-2 \Phi} \ell_{A}^{2}=p_{R}^{3} \sqrt{\frac{\left|q_{0}\right|}{p_{L}^{3}}}
$$


This expression agrees with the result previously found directly in four dimensions (for a good review see [13] 10 ).

We can also determine the $4 \mathrm{D}$ scalars. We are considering the simple situation with $q_{I \neq 0}=0$ and $p^{0}=0$, where there are no M2-branes wrapping the 2-cycles of the CY, nor any magnetic charge of the Kaluza-Klein gauge field from reduction along $x^{5}$. In this case the $4 \mathrm{D}$ scalars other than the dilaton $(6.14)$ are purely imaginary 11 Combining the $5 \mathrm{D}$ scalars (6.10) with the dilaton we find [33]

$$
z^{I}=i e^{-2 \Phi} X^{I}=i p \sqrt{\frac{\left|q_{0}\right|}{p_{L}^{3}}} .
$$

This expression also agrees with results previously found directly in four dimension 12 . Note that in $4 \mathrm{D}$ the $p_{L}$ introduced in (6.12) controls both the scalars and the thermodynamics.

From the $5 \mathrm{D}$ point of view the charge $q_{0}$ corresponds to $\mathrm{AdS}_{3}$ angular momentum. In a two-derivative theory the angular momentum can be read off from the metric via

$$
j=\frac{\rho_{\text {hor }}^{2}}{4 G_{3} \ell_{A}} .
$$

Applying this formula to the corrected metric yields (in our units $G_{3}=\frac{1}{4 \ell_{A}^{2}}$ )

$$
j=\frac{p_{R}^{3}}{p_{L}^{3}}\left|q_{0}\right| .
$$

The mismatch between $j$ and $q_{0}$ is due to the fact that the expressions for conserved quantities as surface integrals themselves receive corrections from the higher derivative terms. It would be instructive to derive these corrected expressions, as was done in [34] for the gravitational Chern-Simons term.

In this section we have focussed on extremal black holes with $T_{L} \neq 0$ and $T_{R}=0$. However, one of the nice features of the $\mathrm{AdS}_{3}$ framework is that our $5 \mathrm{D}$ results easily extend to the non-extremal case $T_{L, R} \neq 0$. The 5D attractor formulas are unchanged, and the general entropy formula is given in (2.6). So higher derivative corrections to the entropy are under control even for these non-BPS, non-extremal black holes [3].

10 The $A d S_{2}$ radius is the $4 \mathrm{D}$ central charge so $R_{0}^{2}=|Z|^{2}$. Combining (6.16) and (6.17) in [13] gives our (6.15) after notation has been adapted.

11 M2-brane charges $q_{I}$ are easily incorporated, as they correspond to Wilson lines for the gauge fields: $w^{I} \sim \frac{1}{R} A_{5}^{I} \sim c^{I J} q_{J}$, with $c^{I J}$ being the inverse of $c_{I J K} p^{K}$.

12 Adapting the notation of (6.18) in [13] gives our (6.16) 
Another feature of the 5D setup is that we can make contact with higher derivative corrections to black rings [35]. Black rings have a near horizon $\mathrm{AdS}_{3} \times S^{2}$ region of the same type as studied here. Therefore our results for the corrected attractor geometry will also apply to the near horizon region of black rings. In particular, it should be possible to find explicit solutions for "small black rings".

\section{Acknowledgments:}

We thank K. Hanaki for discussions. Work of PK and JD supported in part by NSF grant PHY-0456200. The work of FL and AC is supported by DOE under grant DE-FG0295ER40899. 


\section{References}

[1] K. Behrndt, G. Lopes Cardoso, B. de Wit, D. Lust, T. Mohaupt and W. A. Sabra, "Higher-order black-hole solutions in $\mathrm{N}=2$ supergravity and Calabi-Yau string backgrounds," Phys. Lett. B 429, 289 (1998) arXiv:hep-th/9801081; arXiv:hepth/0012232]; "Stationary BPS solutions in $\mathrm{N}=2$ supergravity with $R^{2}$ interactions", JHEP 0012, 019 (2000) arXiv:hep-th/0009234; "Macroscopic entropy formulae and non-holomorphic corrections for supersymmetric black holes", Nucl. Phys. B 567, 87 (2000) arXiv:hep-th/9906094; "Corrections to macroscopic supersymmetric blackhole entropy", Phys. Lett. B 451, 309 (1999) arXiv:hep-th/9812082].

[2] A. Sen, "How does a fundamental string stretch its horizon?," JHEP 0505, 059 (2005) arXiv:hep-th/0411255; "Black holes, elementary strings and holomorphic anomaly," JHEP 0507, 063 (2005) arXiv:hep-th/0502126; "Stretching the horizon of a higher dimensional small black hole," JHEP 0507, 073 (2005) arXiv:hep-th/0505122; "Entropy function for heterotic black holes," JHEP 0603, 008 (2006) arXiv:hepth/0508042] ; B. Sahoo and A. Sen, "alpha' corrections to extremal dyonic black holes in heterotic string theory," JHEP 0701, 010 (2007) arXiv:hep-th/0608182.

[3] P. Kraus and F. Larsen, "Microscopic black hole entropy in theories with higher derivatives," JHEP 0509, 034 (2005) arXiv:hep-th/0506176.

[4] A. Sen, "Black hole entropy function and the attractor mechanism in higher derivative gravity," JHEP 0509, 038 (2005) arXiv:hep-th/0506177.

[5] S. Ferrara, R. Kallosh and A. Strominger, "N=2 extremal black holes", Phys. Rev. D 52, 5412 (1995), [arXiv:hep-th/9508072]; A. Strominger, "Macroscopic Entropy of $N=2$ Extremal Black Holes", Phys. Lett. B 383, 39 (1996), arXiv:hep-th/9602111; S. Ferrara and R. Kallosh, "Supersymmetry and Attractors", Phys. Rev. D 54, 1514 (1996), [arXiv:hep-th/9602136]; "Universality of Supersymmetric Attractors", Phys. Rev. D 54, 1525 (1996), arXiv:hep-th/9603090; R. Kallosh, A. Rajaraman and W. K. Wong, "Supersymmetric rotating black holes and attractors", Phys. Rev. D 55, 3246 (1997), arXiv:hep-th/9611094; A Chou, R. Kallosh, J. Rahmfeld, S. J. Rey, M. Shmakova and W. K. Wong, "Critical points and phase transitions in 5d compactifications of M-theory". Nucl. Phys. B 508, 147 (1997); arXiv:hep-th/9704142.

[6] R. M. Wald, "Black hole entropy is the Noether charge," Phys. Rev. D 48, 3427 (1993) arXiv:gr-qc/9307038. R. Wald, Phys. Rev. D 48 R3427 (1993); V. Iyer and R. M. Wald, "Some properties of Noether charge and a proposal for dynamical black hole entropy," Phys. Rev. D 50, 846 (1994) arXiv:gr-qc/9403028. "A Comparison of Noether charge and Euclidean methods for computing the entropy of stationary black holes," Phys. Rev. D 52, 4430 (1995) arXiv:gr-qc/9503052.

[7] H. Ooguri, A. Strominger and C. Vafa, "Black hole attractors and the topological string", Phys. Rev. D 70, 106007 (2004), arXiv:hep-th/0405146; 
[8] A. Dabholkar, "Exact counting of black hole microstates," Phys. Rev. Lett. 94, 241301 (2005) arXiv:hep-th/0409148.

[9] A. Dabholkar, F. Denef, G. W. Moore and B. Pioline, "Exact and asymptotic degeneracies of small black holes", arXiv:hep-th/0502157; "Precision counting of small black holes," JHEP 0510, 096 (2005) arXiv:hep-th/0507014.

[10] P. Kraus and F. Larsen, "Partition functions and elliptic genera from supergravity," JHEP 0701, 002 (2007) arXiv:hep-th/0607138.

[11] J. de Boer, M. C. N. Cheng, R. Dijkgraaf, J. Manschot and E. Verlinde, "A farey tail for attractor black holes," JHEP 0611, 024 (2006) arXiv:hep-th/0608059].

[12] D. Gaiotto, A. Strominger and X. Yin, "From AdS(3)/CFT(2) to black holes / topological strings," arXiv:hep-th/0602046.

[13] T. Mohaupt, "Black hole entropy, special geometry and strings," Fortsch. Phys. 49, 3 (2001) arXiv:hep-th/0007195.

[14] B. Pioline, "Lectures on on black holes, topological strings and quantum attractors," Class. Quant. Grav. 23, S981 (2006) arXiv:hep-th/0607227.

[15] P. Kraus, "Lectures on black holes and the $\operatorname{AdS}(3) / C F T(2)$ correspondence," arXiv:hepth/0609074].

[16] A. Strominger, "Black hole entropy from near-horizon microstates", JHEP 9802, 009 (1998); arXiv:hep-th/9712251; V. Balasubramanian and F. Larsen, "Near horizon geometry and black holes in four dimensions", Nucl. Phys. B 528, 229 (1998); arXiv:hepth/9802198.

[17] K. Hanaki, K. Ohashi and Y. Tachikawa, "Supersymmetric completion of an $\mathrm{R}^{* *} 2$ term in five-dimensional supergravity," arXiv:hep-th/0611329.

[18] V. Balasubramanian and P. Kraus, "A stress tensor for anti-de Sitter gravity," Commun. Math. Phys. 208, 413 (1999) arXiv:hep-th/9902121.

[19] M. Henningson and K. Skenderis, "The holographic Weyl anomaly," JHEP 9807, 023 (1998) arXiv:hep-th/9806087.

[20] H. Saida and J. Soda, "Statistical entropy of BTZ black hole in higher curvature gravity," Phys. Lett. B 471, 358 (2000) arXiv:gr-qc/9909061.

[21] Y. Tachikawa, "Black hole entropy in the presence of Chern-Simons terms," Class. Quant. Grav. 24, 737 (2007) arXiv:hep-th/0611141.

[22] J. M. Maldacena, A. Strominger and E. Witten, "Black hole entropy in M-theory", JHEP 9712, 002 (1997); arXiv:hep-th/9711053.

[23] J. A. Harvey, R. Minasian and G. W. Moore, "Non-abelian tensor-multiplet anomalies," JHEP 9809, 004 (1998) arXiv:hep-th/9808060.

[24] T. Kugo and K. Ohashi, "Supergravity tensor calculus in 5D from 6D," Prog. Theor. Phys. 104, 835 (2000) arXiv:hep-ph/0006231; T. Fujita and K. Ohashi, "Superconformal tensor calculus in five dimensions," Prog. Theor. Phys. 106, 221 (2001) arXiv:hep-th/0104130. 
[25] E. Bergshoeff, S. Cucu, M. Derix, T. de Wit, R. Halbersma and A. Van Proeyen, "Weyl multiplets of $\mathrm{N}=2$ conformal supergravity in five dimensions," JHEP 0106, 051 (2001) arXiv:hep-th/0104113. "N = 2 supergravity in five dimensions revisited," Class. Quant. Grav. 21, 3015 (2004) [Class. Quant. Grav. 23, 7149 (2006)] arXiv:hepth/0403045].

[26] F. Larsen, "The attractor mechanism in five dimensions," arXiv:hep-th/0608191.

[27] A. H. Chamseddine, S. Ferrara, G. W. Gibbons and R. Kallosh, "Enhancement of supersymmetry near 5d black hole horizon," Phys. Rev. D 55, 3647 (1997) arXiv:hepth/9610155].

[28] J. B. Gutowski and H. S. Reall, "General supersymmetric AdS(5) black holes," JHEP 0404, 048 (2004) arXiv:hep-th/0401129.

[29] A. Dabholkar, A. Sen and S. P. Trivedi, "Black hole microstates and attractor without supersymmetry," JHEP 0701, 096 (2007) arXiv:hep-th/0611143.

[30] J. M. Maldacena and A. Strominger, "AdS(3) black holes and a stringy exclusion principle," JHEP 9812, 005 (1998) arXiv:hep-th/9804085.

[31] M. Banados, M. Henneaux, C. Teitelboim and J. Zanelli, "Geometry of the $(2+1)$ black hole," Phys.Rev.D 66, 010001 (2002) arXiv:gr-qc/9302012.

[32] A. Strominger, "AdS(2) quantum gravity and string theory," JHEP 9901, 007 (1999) arXiv:hep-th/9809027.

[33] K. Behrndt, G. L. Cardoso and S. Mahapatra, "On the relation between BPS solutions in 4D and 5D," Int. J. Mod. Phys. D 15, 1603 (2006).

[34] P. Kraus and F. Larsen, "Holographic gravitational anomalies," JHEP 0601, 022 (2006) arXiv:hep-th/0508218.

[35] I. Bena and P. Kraus, " $\mathrm{R}^{* *} 2$ corrections to black ring entropy," arXiv:hep-th/0506015; N. Iizuka and M. Shigemori, "A note on D1-D5-J system and 5D small black ring," JHEP 0508, 100 (2005) arXiv:hep-th/0506215; A. Dabholkar, N. Iizuka, A. Iqubal and M. Shigemori, "Precision microstate counting of small black rings," Phys. Rev. Lett. 96, 071601 (2006) arXiv:hep-th/0511120]; A. Dabholkar, N. Iizuka, A. Iqubal, A. Sen and M. Shigemori, "Spinning strings as small black rings," arXiv:hepth/0611166]. 